\title{
Cognitive Deficits in Early Parkinson's Disease: New Areas of Research
}

\author{
Sarah L. Mason • Roger A. Barker
}

Published online: 1 February 2012

(C) Springer Science+Business Media, LLC 2012

\begin{abstract}
Cognitive deficits in Parkinson's disease (PD) are heterogeneous, including in the early stages of the disease. The prognostic value of early cognitive deficits is still under debate, but there is particular interest in identifying deficits that are capable of predicting patients at risk of developing dementia. To date, research has focused on four neuropsychological domains (executive/attention, visuo-spatial, memory, and language), but there is recent evidence that impairment may extend beyond this, in particular into the field of social neuroscience. This review will briefly discuss the cognitive impairments in early PD, including the recent evidence for deficits in theory of mind, before evaluating the diagnostic criteria for, and utility of, the term "mild cognitive impairment" in PD.
\end{abstract}

Keywords Parkinson's disease - Cognition .

Neuropsychology · Social cognition · Theory of mind · Mild cognitive impairment $\cdot$ PD-MCI $\cdot$ Premotor

\section{Introduction}

Parkinson's disease (PD) is a common neurological disease that is present in more than $1 \%$ of adults over the age of 65 years, making it the second-most common neurodegenerative disease

S. L. Mason $(\bowtie) \cdot$ R. A. Barker

Cambridge Centre for Brain Repair,

Forvie Site, Robinson Way,

Cambridge CB2 0PY, UK

e-mail: slm64@cam.ac.uk

R. A. Barker

Department of Clinical Neuroscience, Addenbrookes Hospital,

Hills Road,

Cambridge CB2 0QQ, UK after Alzheimer's disease (AD). PD is diagnosed clinically by the presence of bradykinesia, rigidity, resting tremor, and postural instability and pathologically by the presence of alphasynuclein containing Lewy body inclusions in some of the surviving substantia nigra pars compacta neurons [1].

Cognitive impairment is a common and functionally significant problem in $\mathrm{PD}$, with a cumulative prevalence of dementia as high as $75 \%-90 \%$ [2]. Even in the early stages of the disease cognitive changes are detectable [3, 4], with the prevalence of cognitive impairment in nondemented patients estimated to be in the region of $20 \%-40 \%$ [3-6, 7••, 8•]. Deficits are often heterogeneous, occurring in multiple neuropsychological domains, of which the most well-described are impairments of executive function.

Deficits in executive function; the umbrella term used to describe the mechanisms utilized to enable a person to successfully engage in goal-directed behaviors, are thought to represent disruption in the functional integrity of the frontostriatal pathways [9-12]. This pathway is regulated by the transmission of dopamine from one brain region to another. However the role of dopamine in the dysexecutive syndrome found in PD may be a complicated one, with performance on some frontally based cognitive tasks being dependent on disease stage, medication, and the catechol-Omethyltransferase (COMT) genotype of the patient (see [13] for a comprehensive review). This complicated relationship between task performance and dopamine is perhaps best modeled using an inverted U-shaped relationship between executive function and dopaminergic activity in the prefrontal cortex, which proposes that both low and high levels of prefrontal synaptic dopamine can impair performance [14*0]. This also would help explain why many studies have found no relationsip between executive dysfunction in early PD and later dementia [15-17], although not all studies have found this $[6,18,19]$. 
The link between performance on neuropsychological tasks from other cognitive domains and later dementia has been investigated in a number of longitudinal studies with equally conflicting results. Impaired verbal fluency [8•, 20], visuo-spatial deficits [8•], memory [19], and, less frequently, language dysfunction [21] all have been suggested to be indicative of future global cognitive decline, but these findings are limited by inconsistencies in task selection, and patient ascertainment (ie, hospital-based instead of community-based recruitment).

Work characterizing and understanding the basis of these different cognitive deficits in PD is still on-going and has developed now to encompass new cognitive domains such as social neuroscience. This review will briefly evaluate the evidence for distinct cognitive phenotypes in PD before focusing on the current debate surrounding the prognostic value of the cognitive impairment of early PD. In particular, the emerging concept of "mild cognitive impairment" in PD (PD-MCI) and its clinical utility will be evaluated.

\section{The Evolution of Cognitive Phenotypes}

The CamPaIGN study looked at the evolution of cognitive function over time in an incident population of 122 newly diagnosed PD patients recruited from multiple hospital and community sources within the geographical boundary of Cambridgeshire, UK, all of whom were diagnosed with idiopathic PD within a 2 year period [15]. Data at 3.5 and 5.2 years from diagnosis have been published, showing that baseline performance on two neuropsychological tests, namely semantic fluency $(<20$ words in $90 \mathrm{~s})$ and pentagon copying, are significant predictors of dementia independent of age and other potential confounding factors [3, 15]. Suboptimal scores in these neuropsychological predictors plus age greater than 71 years resulted in an odds ratio of $8 / 11$ versus $1 / 34$ patients (OR: $88 ; 95 \%$ CI, $8-962$ ) for the development of dementia at 5.2 years. Performance on semantic and phonemic fluency was dissociable, with only the former found to be associated with later dementia, suggesting that the primary predictive element was the more temporal lobe-based semantic memory component rather than the frontally based search-and-retrieval strategies involved in these tasks. Because no clear deterioration was seen on executive function tasks over this same period of time, the authors concluded that there are two distinct cognitive phenotypes in PD: one consisting of frontostriatal executive deficits that are common but not progressive and another with more posterior cortical deficits, which are indicative of global cognitive decline and eventual dementia. A similar conclusion was reached in a large meta-analysis of 25 longitudinal studies involving 901 PD patients that looked at the differences in the progression of impairment across multiple cognitive domains [17]. This study reported that a significant global cognitive decline was heralded and dominated by deficits in the visuo-spatial function and memory domains, but not in executive function.

Further research into the progression of cognitive decline in PD is needed to definitively determine whether posterior cortical deficits are in fact the precursor or earliest features of the dementia in PD and whether executive dysfunction is a marker for a more benign form of cognitive impairment. However, in light of the findings presented in this review, it would seem that the term PD-MCI should be reserved for the more "malignant" cognitive syndrome, which is predictive of dementia, and not as an umbrella term covering all cognitive impairment in this disease.

\section{Social Cognition in Parkinson's Disease}

The ability to interact effectively in a social situation is a skill necessary for successful communication, a key component of which is the ability to attribute mental states to others. The neural mechanisms underlying these processes have recently become a topic of interest to social cognitive neuroscientists [22-25]. In particular the study of theory of mind (ToM [the ability to understand the presence of beliefs, feelings, intentions, and interests in other people that can differ from their own and from reality]) [26] has migrated from the literature of child development into the field of adult neuropsychology.

ToM is believed to consist of two distinct components: an affective component (belief about feelings) and a cognitive component (belief about belief), and while ToM tasks as a whole have been shown to activate the medial prefrontal cortex (MPFC), temporoparietal junction (TPJ) [27], and amygdala [28], tests of affective ToM have a propensity to activate the ventromedial prefrontal cortex while tasks measuring cognitive ToM tend to preferentially activate more dorsolateral prefrontal cortical areas [29].

ToM deficits have been found in patients with advanced PD [30••, 31-35], which is not entirely unexpected, as both the ventromedial and dorsolateral prefrontal cortex are closely related to subcortical structures involved in PD pathology. In most cases, this deficit was confined to cognitive ToM only [31, 32, 36], with performance on affective ToM tasks being no different from control patients [34-36]. The basis for this deficit in cognitive ToM is not currently known but may relate to changes in frontostriatal dopamine (DA) [37], as:

(1) Neuroanatomically, the regions shown to be involved in ToM are innervated by the dopaminergic and serotonergic (5HT) systems; 
(2) Abnormalities in either of these neurotransmitter systems have been shown to influence cognitive functions such as executive function (as discussed above), which in turn may well contribute to ToM function; and

(3) The dopaminergic system is relevant for signaling predictions about the consequences of future events, an ability that is closely related to ToM.

PD is a good model by which to evaluate the role of dopamine in ToM processing. One study to date has looked at the impact of dopaminergic medication on ToM performance in early PD and reported no effect [38•], with both medicated and unmedicated groups performing equally well on measures of affective ToM and equally badly on cognitive ToM tasks. This would seem to dispute the role of DA in ToM performance; however, the population studied were early PD, although the ToM deficits reported were more in keeping with the impairments found in advance PD patients (see discussion below), indicating that the group may be slightly atypical. Additionally, PD patients were not genotyped for the COMT polymorphism that previously has been shown to affect executive function, and in the medicated group they were assessed in the "on" state. Finally, stage of disease also may be critical; to test this more definitively, an "on/off" study in different stages of disease with genotyped patients is required.

In this respect, ToM deficits have been found in some patients with early PD [38•, 39•, 40-43]. Contrary to the findings in advanced PD, where deficits were only found in cognitive ToM tasks, impairments have been shown on a range of both cognitive and affective ToM tasks in early disease. Santangelo and colleagues [44] found that early PD patients performed worse than control patients on both the Emotion Attribution Task (which measures affective ToM) and the Advance Test of ToM (which measures cognitive ToM) [45]. Performance on the cognitive ToM tasks was associated with neuropsychological performance, as measured by the Frontal Assessment Battery [43] and affective ToM associated with performance on behavioral measures of both frontal behaviors and apathy such as the Frontal Behavioral Inventory [46] and the Apathy Evaluation Scale [47].

The Yoni task is a computerized ToM test that is able to measure both affective and cognitive ToM reasoning within the same test. Participants are asked to evaluate which one of four pictures presented onto a computer screen best corresponds to the simultaneously presented sentence expressing the thoughts of a computerized face, "Yoni" ("Yoni likes ..."; "Yoni is thinking..."). PD patients were found to perform worse than control patients on both the affective and cognitive components of the Yoni task but only for second-order ToM problems [39•] (inferences about "Yoni's" beliefs about another person's belief). Similarly, PD patients were impaired on other ToM tasks measuring both cognitive and affective ToM such as the Cartoons ToM task [40] and the faux pas task [38•], although it is worth noting that others found no difference between early PD patients and control patients on this last task [48•].

The Reading the Mind in the Eyes (RMET) task [41] is a measure of affective ToM that asks participants to judge the thoughts and feelings of another person based only on a picture of the eye region. Both Bodden and colleagues [39•] and Tsuruya and colleagues [49] found that mild to moderate PD patients performed worse than control patients on this task. Additionally, Peron et al. [42] tested 13 PD patients receiving subthalamic nucleus deep brain stimulation pre- and postimplantation and found that PD patients performed significantly worse on the RMET postoperatively when compared to preoperatively or to healthy controls. This correlated with changes in cerebral glucose metabolism in structures known to be involved in the networks that mediate ToM. Post-surgery, all patients were tested with their stimulators "on"; no results were reported from patients whose stimulators had been turned "off," so it is difficult to say whether this is an effect of the surgery or the stimulation.

Not all studies have been able to replicate these findings; Roca et al. [38 $]$ failed to find a difference in performance between early PD patients and control patients on the RMET task; however, this is likely to be for methodological reasons. An abbreviated version of the RMET was used; in this version, 15 stimuli were used instead of the usual 36 and patients were asked to choose from two instead of four adjectives from which to determine the most appropriate description of what the person in the picture was thinking or feeling.

In light of the absence of affective ToM deficits in advanced $P D$, the presence of such impairments in early PD may be indicative that ToM function changes with disease progression in PD. However, without further longitudinal studies, it is impossible to draw any clear conclusions. In addition, the sample sizes in the work published to date have been small ( $n=36$ or below), meaning that it is impossible to assess the heterogeneity of ToM deficits in a PD population. This is necessary to determine whether social cognition has a place alongside the other four recognized cognitive domains (executive/attention, memory, visuo-spatial, and language) that are used to define distinct cognitive phenotypes in PD.

\section{Prognostic Value of Cognitive Deficits in Parkinson's Disease: PD-MCI}

Most longitudinal studies of PD conclude that dementia (PDD) is an inevitable outcome [50-52] if patients are followed up for long enough. The presence of PDD leads 
to significant functional impairment in patients [53-55] and is associated with increased caregiver burden [56, 57], admission into residential care homes [58], and mortality [59]. At present, there are few effective therapeutic options to treat PDD; cholinesterase inhibitors such as rivastigmine are used with only very modest symptomatic effects [60].

Due to the heterogeneous nature of the cognitive deficits in PD, it is difficult to predict when a patient will develop PDD; in some cases, it will be within a few years of diagnosis, but in many others it may be 10 or even 20 years after the emergence of their initial PD symptoms. Being able to identify those patients at immediate risk of significant cognitive decline may dramatically influence the management of their parkinsonism because it would allow clinicians to shift their focus from long-term to shorter-term management strategies as well as the earlier use of drugs that could positively affect cognition, such as cholinesterase inhibitors.

The term mild cognitive impairment (MCI) was originally used to describe the predementia phase in AD that represents a transitional stage between normality and diagnosable disease, characterized by subtle cognitive impairment which results in no or minimal impact on daily functioning [61] although it is being recognized that not all patients with MCI diagnosed in memory clinics go on to develop AD. The label MCI has recently been applied to PD patients with subtle cognitive impairment but who fall short of the diagnostic criteria for dementia.

To date, there is still ambiguity surrounding the diagnostic criteria for PD-MCI, with no universally accepted standard of practice in use, although the Movement Disorders Society is currently in the process of writing such a document. However, as with other such guidelines and the wider MCI literature, cognitive impairment is typically defined by the number of standard deviations (SD) below the ageadjusted mean. However, there is uncertainty about the appropriate number of neuropsychological domains this impairment should be demonstrated in, the number of SDs that should be used as the cut-off, and how this ultimately relates to the development of a subsequent dementia.

A good illustration of this is the study of DalrympleAlford et al. [62••] in a population of 119 nondemented PD patients assessed on 20 neuropsychological measures across four cognitive domains. They showed that the prevalence of PD-MCI could range from 14\% when the conservative criteria of two scores in one domain at two SDs below normal was adopted compared to $89 \%$ of patients (and $70 \%$ of control patients) when a more relaxed criteria of one score from one domain at one SD below normal was used. The authors suggest that two scores below 1.5 SDs from the normative mean, either within one domain (30\% prevalence) or across two domains (37\% prevalence), are the most suitable criteria to adopt.
Aarsland [2] adopted similar criteria to that specified above (1.5 SDs below the normative mean taken as a cutoff for impairment in at least one domain, with test scores within each domain averaged) for a recent large multicenter analysis of 1,341 PD patients, and reported the prevalence of PD-MCI as $25.8 \%$ (CI 23.5-28.2). However, only three cognitive domains were specified (attention/executive, memory, and visuo-spatial) instead of the usual four, and data was not available on all three domains for every site; in addition, at some sites, performance on a domain was dependent on a single neuropsychological test within that domain.

Formalized diagnostic criteria for PD-MCI, which should enable a more consistent approach to the classification and evaluation of early cognitive impairment in PD in future longitudinal studies, are currently under development. However, the prognostic value of PD-MCI, or more specifically, whether all cognitive impairment seen in PD should be classified as PD-MCI is still a contentious issue. As discussed above, it is recognized that cognitive function will decline over time leading to dementia within a relatively short period in some PD patients, while in others deficits may appear early in the disease but remain unchanged or actually even improve over time [52]. As such, the definition of PD-MCI should take this into account, with careful consideration given to the need for subtypes of PD-MCI to be defined in future longitudinal studies.

\section{Conclusions}

Early cognitive impairment is a significant feature in many PD patients and can have a profound effect on quality of life of both patients and caregivers. Work is ongoing into the exact nature of the impairments experienced in these early stages and their relationship to the development of a later dementia. The drawing up of diagnostic criteria for "mild cognitive impairment" in PD is yet to be actualized. Longitudinal data supporting the presence of distinct cognitive phenotypes should be used to inform the clinical application of the term PD-MCI, although further work needs to be done to verify the prognostic dissociation between the dopaminergic frontostriatal dysexecutive syndrome and the more posterior cortically based deficits as we originally proposed [14], as well as their genetic and neurobiological bases.

In addition to the widely documented deficits in the domains of executive function, memory, visuo-spatial processing, and language, there is emerging evidence of impairment in social cognition, in particular ToM. Work in this area is in its infancy and it is still unclear the exact nature of the deficits experienced, their relationship to disease progression or the impact they have on quality of life and daily functioning. However, there are theoretical links between 
ToM function and the dopaminergic frontostriatal network, although this is yet to be confirmed.

Interest in the early and prediagnostic phases of PD is growing, but given the heterogeneous nature of cognitive deficits in PD, there is an urgent need for well-designed longitudinal studies that assess the prognostic value of such impairments. This we have undertaken in part but now requires verification and validation in other cohorts of patients.

Disclosures No potential conflicts of interest relevant to this article were reported.

\section{References}

Papers of published papers of interest have been highlighted as:

- Of importance

•- Of major importance

1. Dickson DW, Braak H, Duda JE, et al. Neuropathological assessment of Parkinson's disease: refining the diagnostic criteria. Lancet Neurol. 2009;8(12):1150-7.

2. Aarsland D. Epidemiology of dementia associated with Parkinson's disease. In: Emre M, editor. Cognitive impairment and dementia in Parkinson's disease. 1st ed. Oxford: Oxford University Press; 2010. p. 5-14.

3. Foltynie T, Brayne CE, Robbins TW, Barker RA. The cognitive ability of an incident cohort of Parkinson's patients in the UK. The CamPaIGN study. Brain. 2004;127(Pt 3):550-60.

4. Muslimovic D, Post B, Speelman JD, Schmand B. Cognitive profile of patients with newly diagnosed Parkinson disease. Neurology. 2005;65(8):1239-45.

5. Caviness JN, Driver-Dunckley E, Connor DJ, et al. Defining mild cognitive impairment in Parkinson's disease. Mov Disord. 2007;22 (9):1272-7.

6. Janvin C, Aarsland D, Larsen JP, Hugdahl K. Neuropsychological profile of patients with Parkinson's disease without dementia. Dement Geriatr Cogn Disord. 2003;15(3):126-31.

7. •- Aarsland D, Bronnick K, Larsen JP, et al. Cognitive impairment in incident, untreated Parkinson disease: the Norwegian ParkWest study. Neurology. 2009 Mar 31;72(13):1121-6. This communitybased study in a large cohort of drug-naïve PD patients looks at the relative risk of $P D-M C I$ compared to control patients.

8. - Mamikonyan E, Moberg PJ, Siderowf A, et al. Mild cognitive impairment is common in Parkinson's disease patients with normal Mini-Mental State Examination (MMSE) scores. Parkinsonism Relat Disord. 2009 Mar;15(3):226-31. This is an interesting study showing the impact of insensitive assessment tools, such as the Mini-Mental State Examination, at detecting mild cognitive impairment in a PD population.

9. Bruck A, Kurki T, Kaasinen V, et al. Hippocampal and prefrontal atrophy in patients with early non-demented Parkinson's disease is related to cognitive impairment. J Neurol Neurosurg Psychiatry. 2004;75(10):1467-9.

10. Lewis SJ, Dove A, Robbins TW, et al. Cognitive impairments in early Parkinson's disease are accompanied by reductions in activity in frontostriatal neural circuitry. J Neurosci. 2003;23(15):6351-6.

11. Rinne JO, Portin R, Ruottinen H, et al. Cognitive impairment and the brain dopaminergic system in Parkinson disease: [18F] fluorodopa positron emission tomographic study. Arch Neurol. 2000;57(4):470-5.

12. Cheesman AL, Barker RA, Lewis SJ, et al. Lateralisation of striatal function: evidence from 18F-dopa PET in Parkinson's disease. J Neurol Neurosurg Psychiatry. 2005;76(9):1204-10.

13. Kehagia AA, Barker RA, Robbins TW. Neuropsychological and clinical heterogeneity of cognitive impairment and dementia in patients with Parkinson's disease. Lancet Neurol. Dec;9(12):1200-13.

14. •- Williams-Gray CH, Evans JR, Goris A, et al. The distinct cognitive syndromes of Parkinson's disease: 5 year follow-up of the CamPaIGN cohort. Brain. 2009 Nov;132(Pt 11):2958-69. This is a well-designed and well-conducted study looking at the predictors of cognitive decline in an incident population of PD patients. The impact of genetic subtypes, demographics, and clinical information are all discussed.

15. Williams-Gray $\mathrm{CH}$, Foltynie T, Brayne $\mathrm{CE}$, et al. Evolution of cognitive dysfunction in an incident Parkinson's disease cohort. Brain. 2007;130(Pt 7):1787-98.

16. Pagonabarraga J, Kulisevsky J, Llebaria G, et al. Parkinson's disease-cognitive rating scale: a new cognitive scale specific for Parkinson's disease. Mov Disord. 2008;23(7):998-1005.

17. Muslimovic D, Schmand B, Speelman JD, de Haan RJ. Course of cognitive decline in Parkinson's disease: a meta-analysis. J Int Neuropsychol Soc. 2007;13(6):920-32.

18. Mahieux F, Fenelon G, Flahault A, et al. Neuropsychological prediction of dementia in Parkinson's disease. J Neurol Neurosurg Psychiatry. 1998;64(2):178-83.

19. Levy G, Jacobs DM, Tang MX, et al. Memory and executive function impairment predict dementia in Parkinson's disease. Mov Disord. 2002;17(6):1221-6.

20. Jacobs DM, Marder K, Cote LJ, et al. Neuropsychological characteristics of preclinical dementia in Parkinson's disease. Neurology. 1995;45(9):1691-6.

21. Hobson P, Meara J. Risk and incidence of dementia in a cohort of older subjects with Parkinson's disease in the United Kingdom. Mov Disord. 2004;19(9):1043-9.

22. Adolphs R. Social cognition and the human brain. Trends Cogn Sci. 1999;3(12):469-79.

23. Adolphs R. Cognitive neuroscience of human social behaviour. Nat Rev Neurosci. 2003;4(3):165-78.

24. Blakemore SJ, Winston J, Frith U. Social cognitive neuroscience: where are we heading? Trends Cogn Sci. 2004;8(5):216-22.

25. Ochsner KN, Lieberman MD. The emergence of social cognitive neuroscience. Am Psychol. 2001;56(9):717-34.

26. Premack D, Woodruff G. Does the chimpanzee have a theory of mind? Behav Brain Sci. 1978;1:515-26.

27. Frith U, Frith CD. Development and neurophysiology of mentalizing. Philos Trans R Soc Lond B Biol Sci. 2003;358(1431):459-73.

28. Van Overwalle F, Van den Eede S, Baetens K, Vandekerckhove M. Trait inferences in goal-directed behavior: ERP timing and localization under spontaneous and intentional processing. Soc Cogn Affect Neurosci. 2009;4(2):177-90.

29. Kalbe E, Schlegel M, Sack AT, et al. Dissociating cognitive from affective theory of mind: a TMS study. Cortex. Jun;46(6):769-80.

30. •• Poletti M, Enrici I, Bonuccelli U, Adenzato M. Theory of mind in Parkinson's disease. Behav Brain Res. Jun 1;219(2):342-50. This is a comprehensive review of theory of mind abilities in PD as they are currently understood.

31. Saltzman J, Strauss E, Hunter M, Archibald S. Theory of mind and executive functions in normal human aging and Parkinson's disease. J Int Neuropsychol Soc. 2000;6(7):781-8.

32. Mengelberg A, Siegert RJ. Is theory-of-mind impaired in Parkinson's disease? Cogn Neuropsychiatry. 2003;8(3):191-209.

33. Mimura M, Oeda R, Kawamura M. Impaired decision-making in Parkinson's disease. Parkinsonism Relat Disord. 2006;12(3):169 75. 
34. Euteneuer F, Schaefer F, Stuermer R, et al. Dissociation of decision-making under ambiguity and decision-making under risk in patients with Parkinson's disease: a neuropsychological and psychophysiological study. Neuropsychologia. 2009;47(13):2882-90.

35. Monetta L, Grindrod CM, Pell MD. Irony comprehension and theory of mind deficits in patients with Parkinson's disease. Cortex. 2009;45 (8):972-81.

36. Peron J, Vicente S, Leray E, et al. Are dopaminergic pathways involved in theory of mind? A study in Parkinson's disease. Neuropsychologia. 2009;47(2):406-14.

37. Abu-Akel A. The neurochemical hypothesis of 'theory of mind'. Med Hypotheses. 2003;60(3):382-6.

38. - Roca M, Torralva T, Gleichgerrcht E, et al. Impairments in social cognition in early medicated and unmedicated Parkinson disease. Cogn Behav Neurol : Off J Soc Behav Cogn Neurol. Sep;23 (3):152-8. This is the first study to systematically look at the impact of dopaminergic medication on theory of mind performance in $P D$.

39. • Bodden ME, Mollenhauer B, Trenkwalder C, et al. Affective and cognitive theory of mind in patients with Parkinson's disease. Parkinsonism Relat Disord. Aug;16(7):466-70. This is one of the few studies to look at the impact of theory of mind on quality of life in Parkinson's disease. The authors found no impact of cognitive performance on theory of mind performance.

40. Gallagher HL, Happe F, Brunswick N, et al. Reading the mind in cartoons and stories: an fMRI study of 'theory of mind' in verbal and nonverbal tasks. Neuropsychologia. 2000;38(1):11-21.

41. Baron-Cohen S, Wheelwright S, Hill J, et al. The "Reading the Mind in the Eyes" Test revised version: a study with normal adults, and adults with Asperger syndrome or high-functioning autism. J Child Psychol Psychiatry. 2001;42(2):241-51.

42. Peron J, Le Jeune F, Haegelen C, et al. Subthalamic nucleus stimulation affects theory of mind network: a PET study in Parkinson's disease. PLoS One. 5(3):e9919.

43. Dubois B, Slachevsky A, Litvan I, Pillon B. The FAB: a Frontal Assessment Battery at bedside. Neurology. 2000;55(11):1621-6.

44. Santangelo G, Vitale C, Trojano L, et al. Neuropsychological correlates of theory of mind in patients with early Parkinson's disease. Mov Disord. Sep 13.

45. Happe FG. An advanced test of theory of mind: understanding of story characters' thoughts and feelings by able autistic, mentally handicapped, and normal children and adults. J Autism Dev Disord. 1994;24(2):129-54.

46. Kertesz A, Davidson W, Fox H. Frontal behavioral inventory: diagnostic criteria for frontal lobe dementia. Can J Neurol Sci. 1997;24(1):29-36.

47. Marin RS, Biedrzycki RC, Firinciogullari S. Reliability and validity of the Apathy Evaluation Scale. Psychiatry Res. 1991;38(2):143-62.
48. - Yu RL, Wu RM, Chiu MJ, et al. Advanced theory of mind in patients at early stage of Parkinson's disease. Parkinsonism Relat Disord. Aug 23. This is an interesting study looking at performance on advanced theory of mind tasks in early PD patients.

49. Tsuruya N, Kobayakawa M, Kawamura M. Is "reading mind in the eyes" impaired in Parkinson's disease? Parkinsonism Relat Disord. May;17(4):246-8.

50. Buter TC, van den Hout A, Matthews FE, et al. Dementia and survival in Parkinson disease: a 12-year population study. Neurology. 2008;70(13):1017-22.

51. Hely MA, Reid WG, Adena MA, et al. The Sydney multicenter study of Parkinson's disease: the inevitability of dementia at 20 years. Mov Disord. 2008;23(6):837-44.

52. Aarsland D, Andersen K, Larsen JP, et al. Prevalence and characteristics of dementia in Parkinson disease: an 8-year prospective study. Arch Neurol. 2003;60(3):387-92.

53. Bronnick K, Ehrt U, Emre M, et al. Attentional deficits affect activities of daily living in dementia-associated with Parkinson's disease. J Neurol Neurosurg Psychiatry. 2006;77(10):1136-42.

54. Rosenthal E, Brennan L, Xie S, et al. Association between cognition and function in patients with Parkinson disease with and without dementia. Mov Disord. Jul 15;25(9):1170-6.

55. Uc EY, Rizzo M, Anderson SW, et al. Driving with distraction in Parkinson disease. Neurology. 2006;67(10):1774-80.

56. Aarsland D, Larsen JP, Karlsen K, et al. Mental symptoms in Parkinson's disease are important contributors to caregiver distress. Int J Geriatr Psychiatry. 1999;14(10):866-74.

57. Schrag A, Hovris A, Morley D, et al. Caregiver-burden in Parkinson's disease is closely associated with psychiatric symptoms, falls, and disability. Parkinsonism Relat Disord. 2006;12(1):35-41.

58. Aarsland D, Larsen JP, Tandberg E, Laake K. Predictors of nursing home placement in Parkinson's disease: a population-based, prospective study. J Am Geriatr Soc. 2000;48(8):938-42.

59. Aarsland D, Bronnick K, Ehrt U, et al. Neuropsychiatric symptoms in patients with Parkinson's disease and dementia: frequency, profile and associated care giver stress. J Neurol Neurosurg Psychiatry. 2007;78(1):36-42.

60. Emre M, Aarsland D, Albanese A, et al. Rivastigmine for dementia associated with Parkinson's disease. N Engl J Med. 2004;351 (24):2509-18.

61. Petersen RC. Mild cognitive impairment as a diagnostic entity. J Intern Med. 2004;256(3):183-94.

62. • Dalrymple-Alford JC, Livingston L, Macaskill MR, et al. Characterizing mild cognitive impairment in Parkinson's disease. Mov Disord. $2011 \mathrm{Feb}$ 1. This is an important paper looking at the impact of different diagnostic criteria of PD-MCI on the variation in the proportion of cases identified. 